\title{
Brain Image Analysis, Classification and Reconstruction
}

\author{
Andrew Karam \\ Computer Science department \\ Faculty of Computer Science and \\ artificial intelligence, Helwan \\ University, Cairo, Egypt
}

\author{
Mario Rouf \\ Computer Science department \\ Faculty of Computer Science and \\ artificial intelligence, Helwan \\ University, Cairo, Egypt
}

\author{
Kirollos Boles \\ Computer Science department \\ Faculty of Computer Science and \\ artificial intelligence, Helwan \\ University, Cairo, Egypt \\ Mina Atef \\ Computer Science department \\ Faculty of Computer Science and \\ artificial intelligence, Helwan \\ University, Cairo, Egypt \\ Minaatef@fci.helwan.edu.eg
}

\author{
Kirollos Samer \\ Computer Science department \\ Faculty of Computer Science and \\ artificial intelligence, Helwan \\ University, Cairo, Egypt \\ Ghada A. khoriba \\ Computer Science department \\ Faculty of Computer Science and \\ artificial intelligence, Helwan \\ University, Cairo, Egypt
}

\section{ABSTRACT}

Decoding brain activity occurs in response to an external stimulus is a great problem and the first step to analysis, classification, reconstruction of the external stimulus which will lead to some marvelous application like reconstructing the visual images from the mind of a person in comma or identifying an object in the head of someone that has some psychological problem in the medical field, and criminal image reconstruction instead of forensic artists in security problems or instant design in marketing, etc...

The problem with brain data analysis is that the brain data like fMRI, which we used here, is very complicated. Also acquiring the data is difficult and requires a lot of resources and time and this is why the fMRI datasets size is small. The data is divided into 3 subjects and each subject data consists of fMRI responses to 3 types of images (letters, artificial, natural). In this work, we focused on 3 main targets: analysis and classification of the difference between subjects and types of images, identification of objects presented in the natural images, and finally reconstruction of the images from fMRI data.

\section{INTRODUCTION}

Brain activities occur when our brains are triggered. Decoding those activities to learn about the triggers is a great challenge because the brain is complicated. A particular challenge is the analysis of brain activities of visual stimulus images. The goal of the analysis is to learn, classify, and reconstruct the visual stimuli images. Those leading to applications can assist in many fields. This work focused on decoding the brain activities of visual stimulus images from the visual cortex, by working with fMRI.

This work focuses on analyzing the fMRI data, classifying the image type (Letter, Artificial shape, Natural images), and reconstruction of the visual image. The research worked with fMRI data Kamitani-Lab[1], the data consists of subjects (per-sons) every subject has sessions, every session contains runs, the run can be one of three types which the subject watches it (Letter, Artificial, Natural Images).
In this research, Deep Neural Network CNN and machine learning classifier SVM be used for classification the types with accuracy approximately $99.5 \%$ for $\mathrm{CNN}$ and $88 \%$ for SVM. For classifying the type of images, using a specific region of interest (ROI) got approximately $98.75 \%$ for CNN and $40.5 \%$ for SVM, and for classification between the colors of the artificial shapes got $45 \%$ using SVM.

Moreover, for more classification (The Natural category), first, by following Tomoyasu Horikawa and Yukiyasu Kamitani[2] extract features from stimulus images using the CNN model. Then, decoded fMRI to features of a visual image using different regression models and compare the result. Finally, the decoded features were compared to identify the most similar category.

For image reconstruction, we followed K.Seeliger et. al. [3]. Deep convolutional Generative Adversarial Networks (DCGAN) were trained to generate natural images. In another site, the linear model was learned to predict the earned distribution conventionally called latent space $\mathrm{z}$ from the fMRI activity. Then the output of the linear regression is forwarded to the DCGAN as input and the generated image compared with the original image using a feature-matching model to optimize the linear model. The DCGAN reconstructed the stimulus image from the input $\mathrm{z}$.

\section{Proposed model}

We provide an illustration for fMRI, details about the dataset, and the result of the analysis, classification, and identification.

The proposed model consists of three steps: the first is visualization of the data; the second is classification, then reconstruction of the visual images.

a) Visualization:

The data consists of subjects (person), each subject has a session. The subject watches stimulus images (Artificial, Letter, and Natural Images), every stimulus image in this time affects the brain. So we assume that each subject has a specific effect on his brain according to the type of image 
(hypothesis 1), because natural images have many details more than artificial images and artificial images more than letter images. Also, we assumed that natural images have more effect on the brain (hypothesis 2). By applying some statistical measures for proving our hypothesis, this statistical measure is the mean, max, and sum of each voxel's intensity.

\section{b) Classification:}

For proving the hypotheses 1 and 2, we used classification models. By using Support Vector Machine SVM and CNN ${ }^{1}$, for classifying between the types of images. The result of proving the hypotheses gave us another hypothesis that the color can affect the brain with a particular effect, so we used the classification model to classify the color of each artificial shape (Hypothesis 3). The proven hypothesis is given more thinking that the brain effect with particular distribution with the category of each natural image (Hypothesis 4). The categories of the natural images (200 categories) were classified using CNN with the two mask approaches, but the result is not good. The classification between the natural categories failed because of many reasons, one of them is the number of categories big compared to the data for each category.

So, we used another approach to classify the category of each image in the natural images (200 categories) by following Tomoyasu Horikawa and Yukiyasu Kamitani [2]. They decode the categories by providing a decoding model. First, using the CNN model to extract features of images. Second, using a regression model to relate the features with fMRI data as a decoder. Then provide a model to predict the most similar categories. They used sparse linear regression as a decoder, but we used ridge regression [6] instead of sparse regression. The reason for choosing ridge regression, the features of the data are more than the number of observations and this reason leading the sparse linear regression to overfit..

\section{c) Reconstruction}

From the proven hypotheses 1, 2, 3, and 4, we assume another hypothesis that every image has a particular effect on the brain and it is a good intuition for the reconstruction of the visual images (Hypothesis 4). For reconstructing the visual image, we followed K. Seeliger et. al.[3], this approach used DCGAN which is a network trained to generate the image based on the distribution (latent space learned from the training data). Then use linear regression with input fMRI data and stimulus images as a label to predict latent space then pass to the generator model. This research provides reconstructing color and gray images, unlike K. Seeliger et. al.[3] provided reconstructing gray images.

\section{Results and Discussion}

This section provides the result and the materials on it, by proving the assumed hypotheses.

\section{Materials}

\section{- Dataset:}

The fMRI dataset ${ }^{2}$ consisted of 3 subjects. Each subject has sessions. Every session contains a run, the run can be one of three types which the subject watch it (Letter,
Artificial, Natural Images). Also, anatomy session that aimed to capture the brain structure of the subject without introducing any stimulus. Letters consisted of 10 images, Artificial: 40 images, Natural test: 50 categories x 1 images for each (50 images), Natural train: 150 categories x8 images for each (1200 images). The runs are where the fMRI brain activity data was captured. By presenting a stimulus to the subject and capture, the fMRI images in certain time-points.

\section{- Masks:}

The MRI data represented as a 3D matrix consists of the position of the voxel ${ }^{3}$ and its value/intensity. Each MRI represents the whole brain at a time point. The brain consists of the cortex (parts). This study focused on the visual cortex only, so it was necessary to extract voxels of the visual cortex-x and ignore the rest. Specific regions of the visual cortex are more affected (Regions Of Interest [4][5]) . It was necessary to mask the fMRI images to obtain the regions of interest (ROI), also for accurate mapping between brain activities and stimulus. ROI differs for each subject. Two masking approaches were used, first: masking each subject separately, and the second: using a combined mask for all subjects.

\section{Results}

Hypothesis 1: Assume that each subject has a specific effect on his brain according to the type of image. By analyzing the data, we found that each image type has a particular distribution in the brain. As shown in Figures [1,2, and 3] each data type (Natural, Artificial, Letter) has a particular effect that confirmed Hypothesis 1. For hypothesis 2 , the natural images have more effect on the brain the figures $[1,2,3]$ prove that.

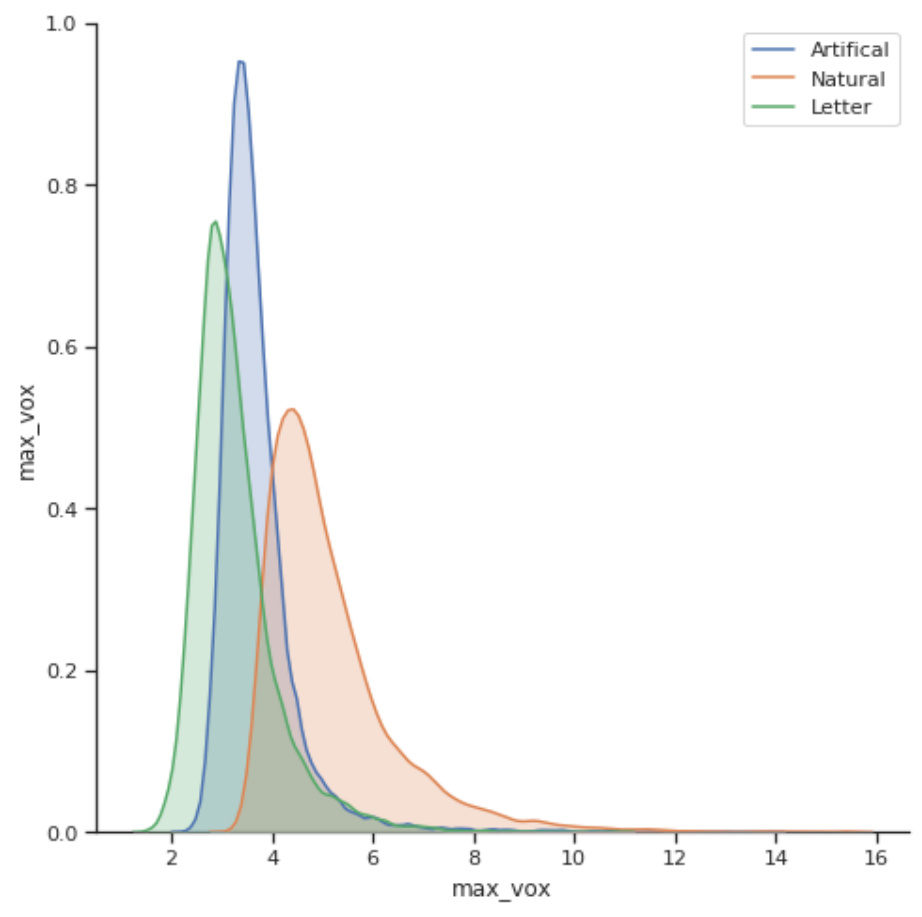

Figure 1. The distribution for each type of images using max statistical measures for subject 1 1 The CNN model consists of 3 blocks inspired from VGG-19 but with freeze
some layers and change the kernel.

\footnotetext{
2 https://openneuro.org/datasets/ds001506/versions/1.3.1

${ }^{3}$ Voxel is a cube that contains many neurons
} 


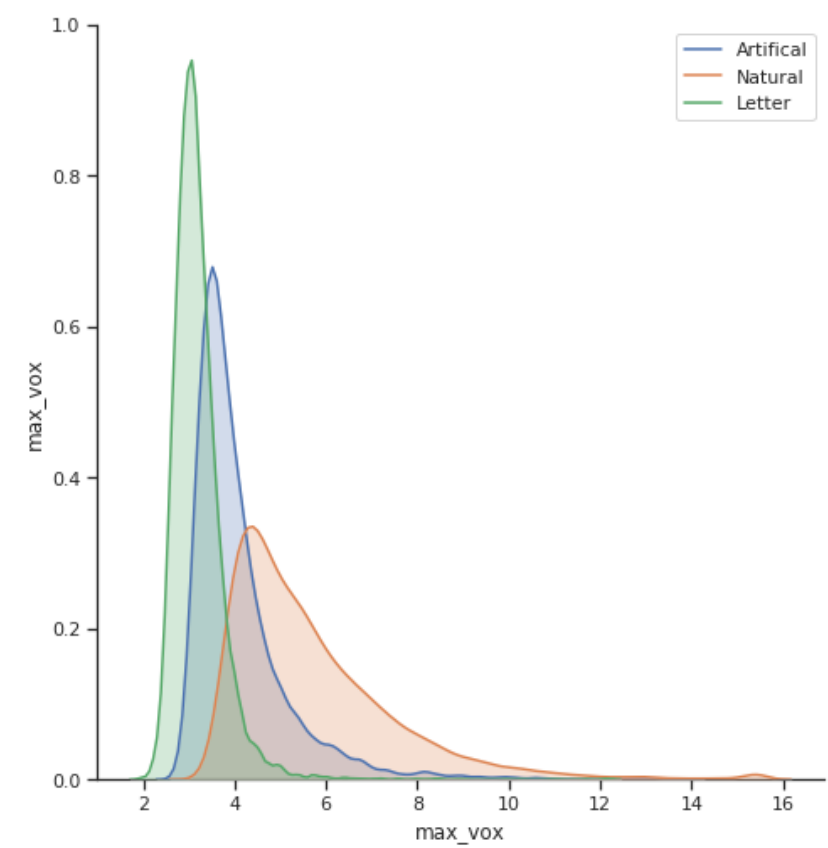

Figure 2. The distribution for each type of images using max statistical measures for subject

Hypothesizes 1 and 2 give us more intuition that we can classify between each type using traditional machine learning and Deep learning CNN. By using SVM for classifying the types of images, the model could classify with accuracy $88 \%$ among the three subjects with the first mask approach. With the second approach of masking, the model could classify with $61.3 \%$ among the three subjects. But with using CNN for the classifying the types of images, got $99.3 \%$ with using masking one, and using masking two the accuracy is $90.3 \%$. The classification result provides more proof for hypotheses 1 and 2 .

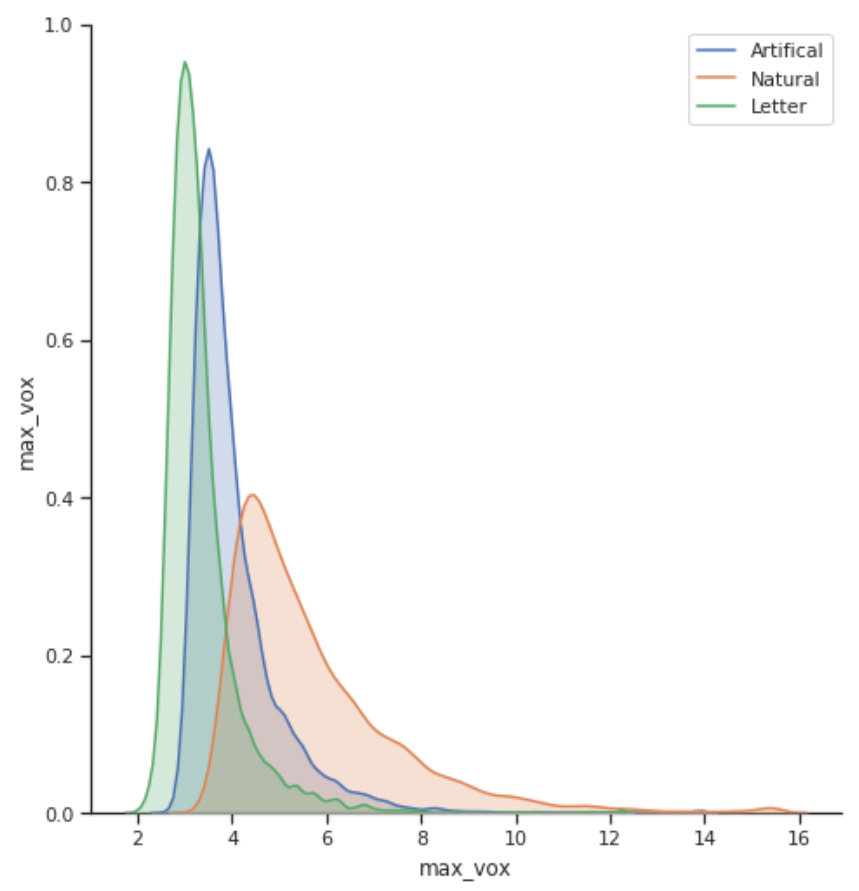

Figure 3. The distribution for each type of images using max statistical measures for subject 3
Hypothesis 3: Assume that our brain can be affected by a specific distribution when our brain is stimulated by a specific color. The artificial images are 40 images, the color of artificial shapes in 8 colors (white, black, green, blue, red, yellow, cyan, magenta). By using the color as a category, the result is shown when classifying between RGB (red, green, and blue) the accuracy is $35.83 \%$ with the first masking approach and $37.3 \%$ with second masking approach. When classifying bet-ween Blue and cyan, the result is $52 \%$ with first and second masking approaches. With the small data of artificial shapes, the result showed that the models could not classify with good accuracy. If the data increase, the model can classify with good accuracy, so hypothesis 3 till now is not right.

Hypothesis 4: Assume that each natural category images have a particular effect on the brain. For this hypothesis, we tried to classify between the categories of natural images using the CNN model, but got $25 \%$ as bad accuracy. The bad accuracy because the images for each category is not enough to train on it. For this reason, we followed Tomoyasu Horikawa and Yukiyasu Kamitani [2]. As mentioned in the proposed model section, we change the sparse linear regression by Ridge linear regression. The result of the identification model is represented in Table 1; the table shows that the result of sparse linear regression is similar to the ridge regression. The advantage of using ridge regression, the time of training is 42 times less than sparse linear regression.

Table 1. Result of identification the category for each test images using sparse linear and ridge regression. There is no difference in the accuracy the time with ridge regression 42 time in result

\begin{tabular}{|c|c|c|c|}
\hline & $\begin{array}{c}50^{4} \\
\text { Category }\end{array}$ & 1050 Category & 15372 Category \\
\hline Sparse & $82 \%$ & $48 \%$ & $4 \%$ \\
\hline Ridge & $82 \%$ & $46 \%$ & $2 \%$ \\
\hline
\end{tabular}

Hypothesis 5: Assume that each image has a particular effect on our brain. For testing hypothesis 5, reconstruct the visual image and classify the letter images. If the reconstruction and classification success, then the hypothesis is true. The result of reconstruction is shown in figures 4 , and 5. the size of train data for reconstructing visual images is very small because of the limited resources. The result of the classification of the letter images is $63 \%$ with the first masking approach and $66 \%$ with the second masking approach using the $\mathrm{CNN}$ model. The result of reconstruction showed that hypothesis 5 can be true especially if the size of training data increases and the classification result approved that.

\footnotetext{
${ }^{4}$ For choose the closer category, take the max correlation coefficient between the predicted features and the category features. The compared category can be any numbers of category, we try 50 category (test category), 1050 and 15372 .
} 

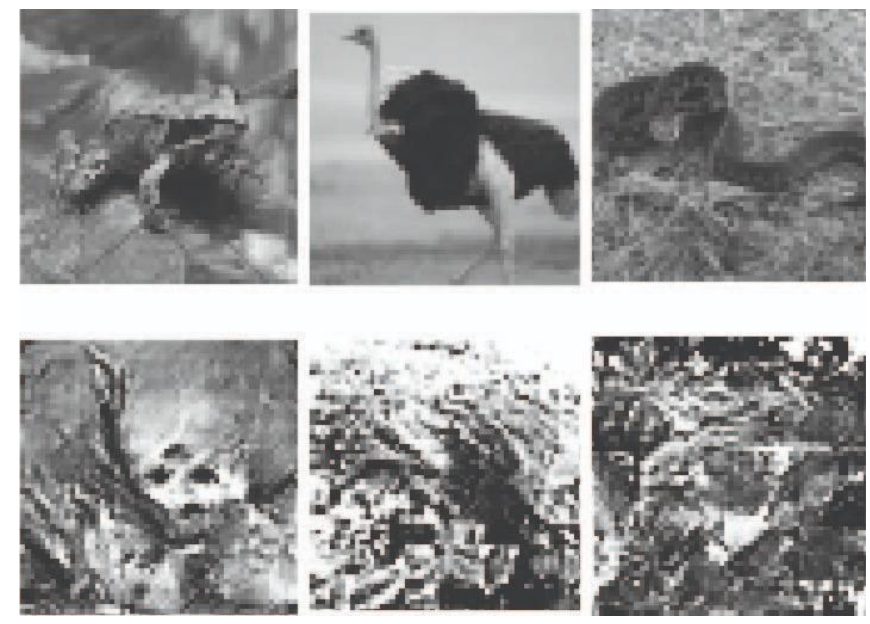

Figure 4. Reconstruction visual images, examples of the reconstructed gray images

\section{Conclusions}

Human brain is very complicated, and extracting meaningful information is not easy. The brain has many areas, each of them is responsible for something (heard. feeling, etc.), and one of them is responsible for the visual cortex (VC) which is used. The brain is affected when it is stimulated by outside or in-side effects, so we assume some of the hypotheses. We assumed that the brain is affected by a particular effect (distribution) when the person watches the type of data instead of another type. Also, we assumed that the natural images have more effect on the brain more than artificial images and artificial shapes more than letters. The result of analysis using statistical measures and classification using the CNN model and SVM showed that the brain has more effect when the brain is exposed to more detailed images. The proven hypothesis let us hypothesize that the brain is affected by a particular effect when the person watches with a specific color. The classification using the SVM model for artificial images shows that the brain can have a particular distribution for each color with litt-le accuracy (probably the little accuracy because of the small size of artificial images). For another hypothesis that the brain is affected with specific distribution by the category of natural images (200 categories). By using the CNN model, the model could not classify the natural category, so using an identical approach to identify the natural images with features of the category of the natural images and the testing image. The main goal of the research (Reconstruction the visual images), the hypothesis term for this part that every image has a particular effect. For this hypothesis, train a CNN model to classify between the letter images and reconstruct the natural images. The result of the classification model and the reconstruction model showed that the hypothesis could be true when the size of the data increases.
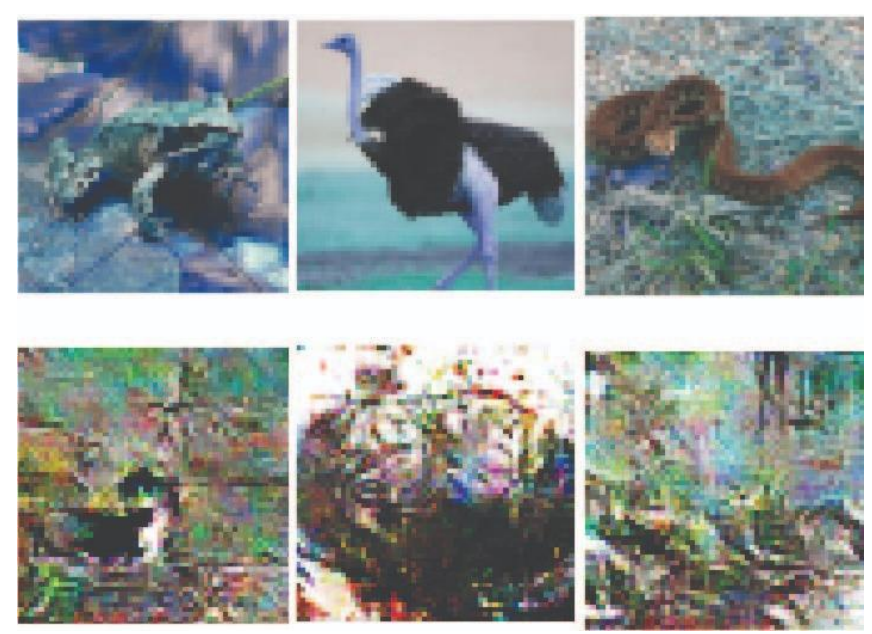

Figure 5. Reconstruction visual images, examples of the reconstructed color images

\section{Recommendations}

This section provides some recommendations when working on medical data sets.

1. If you are working on medical data fMRI or another, you should mask your data set to ignore the rest area and take only the region of interest (ROI)

2. If the features of the data set are bigger than the observations, and you are working with regression models. You have to try ridge or lasso regression because of the shrinkage.

\section{REFERENCES}

[1] KamitaniLab. fmridata.

https://openneuro.org/datasets/ds001506/versions/1.3.0/download.

[2] Tomoyasu Horikawa and Yukiyasu Kamitani. Generic decoding of seen and imagined objects using hierarchical visual features.Nature Communications, 8(1):15037, 2017.

[3] K.Seeliger, UmutG ü lı̈u,L.Ambrogioni,Ya gmur Güuc lüü urk, and M.A.J. Gerven.Generative adversarial networks for reconstructing natural images from brain activity.NeuroImage, 181, 072018

[4] G. Shen, T. Horikawa, K. Majima, and Y. Kamitani, "Deep image reconstruction from human brain activity,"PLOS Computational Biology, vol. 15, pp. 1-23, 01 2019.

[5] M. T. Tomoyasu Horikawa, Shuntaro C. Aoki and Y. Kamitani, Characterization of deep neural network features by decodability from human brain activity, pp. 75-85. Scientific Data, 12 February 2019.

[6] Donald Marquardt and Ron Snee.Ridge regression inpractice.American Statistician - AMER STATIST, 29:3-20,02 1975 